\title{
HIGH INTENSITY HEAVY ION BEAM EMITTANCE MEASUREMENTS AT THE GSI UNILAC
}

\author{
W. Bayer*, W. Barth, L. Dahl, P. Forck, P. Gerhard, L. Groening, I. Hofmann, \\ S. Yaramyshev, GSI, D-64291 Darmstadt, Germany, \\ D. Jeon, ORNL, Oak Ridge, TN37830, USA
}

\section{Abstract}

The GSI UNILAC, a heavy ion linac originally dedicated for low current beam operation, together with the synchrotron SIS 18 will serve as a high current injector for FAIR (International Facility for Antiproton and Ion Research). The UNILAC post stripper accelerator consists of five Alvarez tanks with a final energy of $11.4 \mathrm{MeV} / \mathrm{u}$. In order to meet the requirements of FAIR $\left(15 \mathrm{emA}{ }^{238} \mathrm{U}^{28+}\right.$, transverse normalised emittances of $0.8 \mathrm{~m} \mathrm{mrad}$ and $2.5 \mathrm{~mm}$ mrad an UNILAC upgrade program is foreseen to increase the primary beam intensity as well as the beam brilliance. A detailed understanding of the beam dynamics during acceleration and the transport of space charge dominated beams is necessary. For this purpose the beam brilliance dependency on the phase advances in the Alvarez DTL was studied. Machine investigations were performed with various beam diagnostics devices established in the UNILAC. Measurements done in 2006 using a high intensity heavy ion beam coincide with the beam dynamics work package of the European JRA "High Intensity Pulsed Proton Injector" (HIPPI). Results of these measurements are presented as well as corresponding beam dynamics simulations.

\section{INTRODUCTION}

The GSI heavy ion accelerator complex consists presently of the UNIversal LInear ACcelerator UNILAC (Figure 1) and the heavy ion synchrotron SIS 18. Heavy ion beams are delivered to the High Current Injector (HSI) from two different ion source terminals: Penning type (PIG) sources for low and MUCIS/ MEVVA type sources for high beam intensities. An RFQ and two IH structures accelerate the ions from $2.2 \mathrm{keV} / \mathrm{u}$ up to $1.4 \mathrm{MeV} / \mathrm{u}$. Afterwards the charge state is increased by a gas stripper and a subsequent magnetic charge separator system. Additionally an ECR source and the High Charge Injector (HLI) provide for directly high charged particle beams. The beam is matched to the Alvarez Drift Tube Linac (DTL) by two rebunchers and five quadrupoles and then accelerated up to $11.4 \mathrm{MeV} / \mathrm{u}$. In the transfer channel (TK) to SIS 18 a stripper foil optionally provides for beams of higher charge states.

For the future accelerator complex of FAIR the UNILAC has to deliver a ${ }^{238} \mathrm{U}^{28+}$ beam to SIS 18 with up to $15 \mathrm{emA}$ and normalised transverse emittances of $\beta \gamma \epsilon_{\mathrm{x}}=$ $0.8 \mathrm{~mm} \mathrm{mrad}$ and $\beta \gamma \epsilon_{\mathrm{y}}=2.5 \mathrm{~mm} \operatorname{mrad}$ [1]. As the Alvarez quadrupole power supplies do not provide for adequate phase advances for high intense heavy ion beams [2],

* email: w.bayer@gsi.de

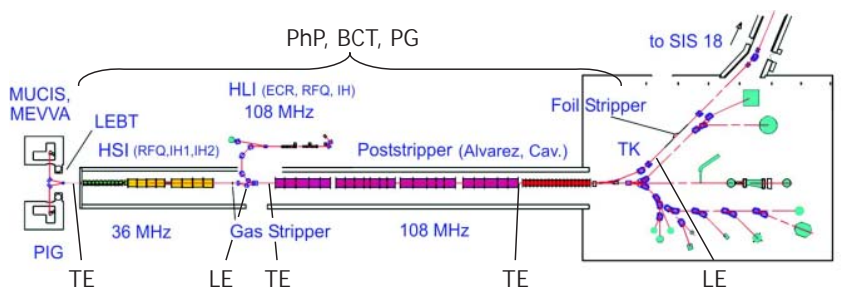

Figure 1: Schematic overview of the GSI UNILAC and important beam diagnostics: transverse (TE) and longitudinal (LE) emittance measurement devices, phase probes $(\mathrm{PhP})$, beam current transformers (BCT) and profile grids (PG).

it is assumed, based on calculations with the multiparticle simulation code PARMILA, that just $8.4 \mathrm{emA}$ of ${ }^{238} \mathrm{U}^{28+}$ are contained within these emittances.

An upgrade program for the UNILAC is still in progress to meet the requirements for FAIR. To prepare the measures of the upgrade, dedicated machine experiments as well as attendant simulations are mandatory.

Within the beam dynamics work package of the European JRA "High Intensity Pulsed Proton Injector" (HIPPI) benchmarking of different beam dynamics simulation codes is performed as well as a comparison to measurements. In consideration of both tasks for the first time exclusive beam time ( 96 shifts) for UNILAC machine experiments was requested and approved for 2006 by the GSI Program Advisory Committee.

\section{EXPERIMENTAL SETUP}

For high beam intensity machine experiments important beam diagnostic devices (see Figure 2) are located inbetween the gas stripper and the Alvarez DTL, that are devices for the measurement of the longitudinal $[3,4]$ and the transverse emittances (slit/grid type). For the determination of the transverse emittance growth along the DTL the slit/grid device behind the DTL was used. Further phase probes, beam current transformers, and profile grids are installed along the UNILAC.

All investigations were performed with a $7.1 \mathrm{emA}$ ${ }^{40} \mathrm{Ar}^{10+}$ beam, which is space charge equivalent to $15 \mathrm{emA}$ ${ }^{238} \mathbf{U}^{28+}$. Due to the lower mass over charge ratio $(\mathrm{m} / \zeta)$ of ${ }^{40} \mathrm{Ar}^{10+}$ the maximum focusing strength of the Alvarez

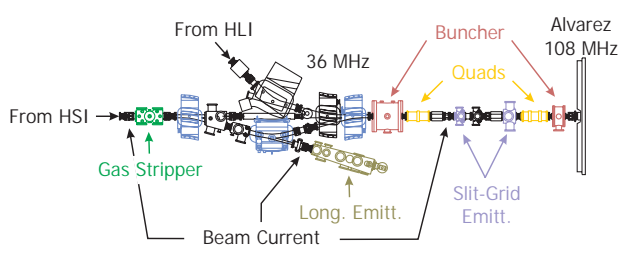

Figure 2: Schematic overview of the gas stripper including the beam diagnostic devices. 
quadrupoles is more than twice larger.

\section{MATCHING TO THE ALVAREZ DTL}

Beside the optimisation of HSI and Alvarez DTL settings, concerning the transmission and minimisation of energy parasits [5], the matching of a space charge affected beam to the DTL is of major interest to provide for minimised emittance growth during acceleration.

The general matching procedure is described in [6]. To calculate the settings of the two rebunchers and the five quadrupoles (see Figure 2) to match to the periodic solution of DTL tank 1, a routine is used which is based on rms-envelope-tracking considering space charge forces [7]. Measured longitudinal and transverse emittances as well as the rebuncher and quadrupole settings are used as input parameters. The routine minimises the mismatch factor defined by [7] varying these seven parameters. It results in rebuncher and quadrupole settings for a minimum calculated mismatch, the estimated Twiss parameters at DTL entrance, and the periodic solution.

Before the calculated settings are applied to the rebunchers and quadrupoles the DTL transmission and the transverse emittances behind the Alvarez DTL are measured to determine changes in the beam quality, optionally a second matching is performed.

\section{MEASUREMENT RESULTS}

With respect to the UNILAC upgrade a variation of the zero current phase advance $\sigma_{0}$ between $35^{\circ}$ and $90^{\circ}$ was performed to find the $\sigma_{0}$ value for minimum emittance growth along and hence maximum beam brilliance behind the DTL. The following procedure was used:

1. All DTL quadrupoles were set to a selected $\sigma_{0}$.

2. Using the matching routine the rebuncher and quadrupole settings were calculated for optimised matching to the DTL at this $\sigma_{0}$ value.

3. These values were applied except a correction of the rebuncher settings to preserve a transmission close to $100 \%$. The measured input phase space distributions for the matching calculations of all $\sigma_{0}$ values are shown in Figure 3.

4. Finally the transverse emittance behind the Alvarez DTL was measured.

Some examples of measured phase space distributions behind the DTL for different $\sigma_{0}$ are presented in Figure 4.

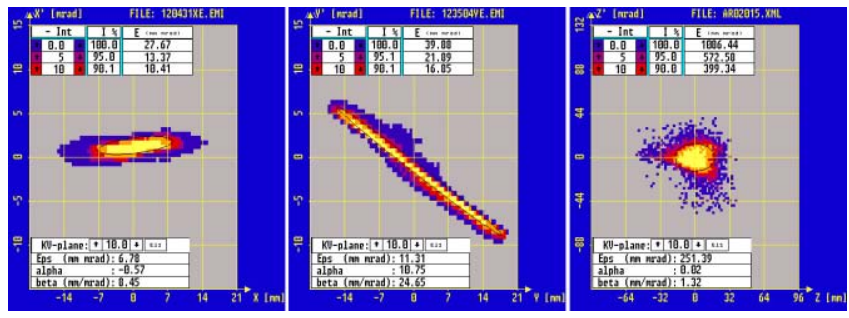

Figure 3: Measured horizontal (left), vertical (middle) and longitudinal (right) phase space distributions before the Alvarez DTL for matching calculations.

04 Hadron Accelerators
Figure 4: Measured transverse beam emittances behind the DTL for different $\sigma_{0}$. The standard operation value is $45^{\circ}$.

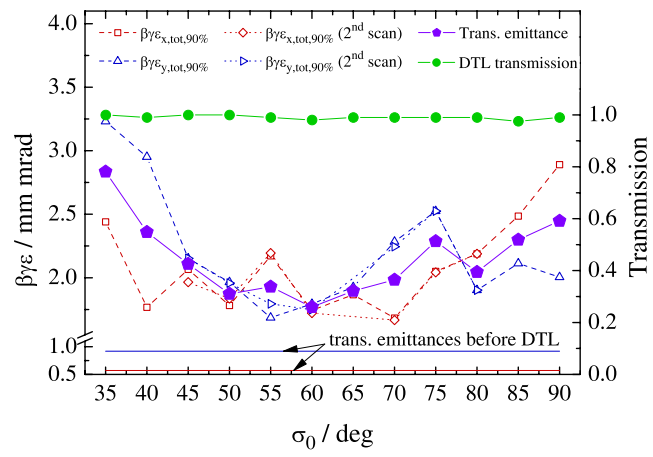

Figure 5: Measured transverse emittances behind the Alvarez DTL as function of $\sigma_{0}$.

Initially the reproducibility of the $\sigma_{0}$ variation procedure as well as the emittance measurement was investigated. The DTL transmission and the transverse emittances behind the DTL were measured in a first scan for phase advance values between $\sigma_{0}=45^{\circ}$ and $\sigma_{0}=80^{\circ}$. The comparison with a second scan performed afterwards (see Figure 5) shows excellent reproducibility within the systematic error of $10 \%$ of each measurement.

Advanced measurements were performed in the complete range from $\sigma_{0}=35^{\circ}$ to $\sigma_{0}=90^{\circ}$ in steps of $5^{\circ}$. The results are shown in Figure 5. For all $\sigma_{0}$ values the transmission is nearly $100 \%$. The minimum emittances in both transverse planes of $\beta \gamma \epsilon_{\mathrm{x}, \text { tot }, 90 \%}=1.74 \mathrm{~mm} \mathrm{mrad}$ and $\beta \gamma \epsilon_{\mathrm{y}, \mathrm{tot}, 90 \%}=1.79 \mathrm{~mm} \mathrm{mrad}$ were observed at $\sigma_{0}=$ $60^{\circ}$. This corresponds to a mean transverse emittance growth of 2.15 , i.e. the beam brilliance was increased about $20 \%$ in comparison to the standard phase advance of $\sigma_{0}=45^{\circ}$. Thus, about $70 \%$ of the beam intensity delivered by the UNILAC has been inside the acceptance of SIS 18.

\section{SIMULATION RESULTS}

In the framework of HIPPI the measurement results of the $\sigma_{0}$ variation were compared with simulation results of the beam dynamics codes DYNAMION [8] and PARMILA [9]. For both codes the settings of the rebunchers and quadrupoles in the matching section as well as the transverse emittances before the Alvarez DTL were used as input parameters. Introducing all measured longitudinal 

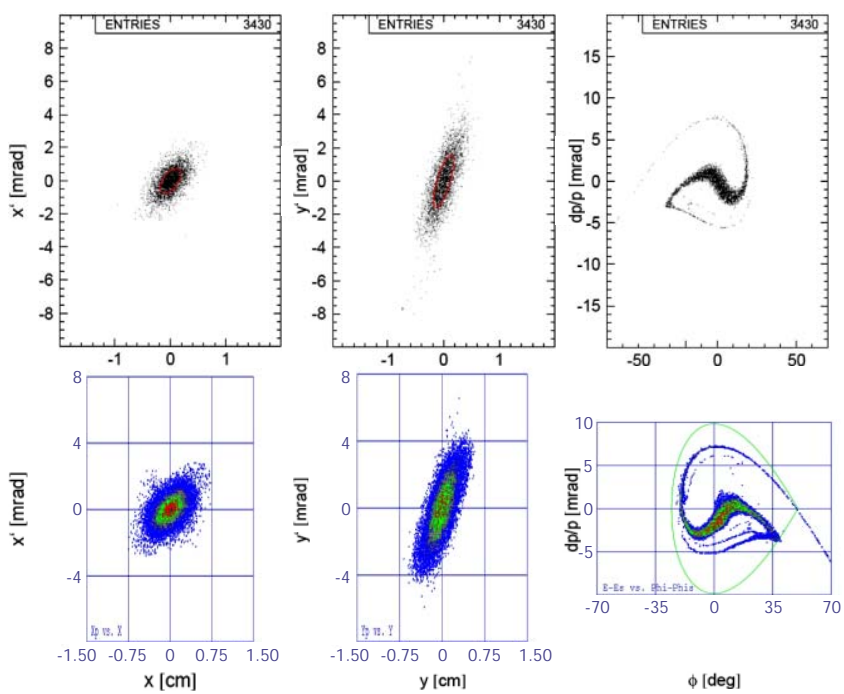

Figure 6: Phase space distributions behind DTL at $\sigma_{0}=60^{\circ}$ simulated with DYNAMION (top) and PARMILA (bottom). The longitudinal distributions are mirror-inverted as $\phi_{\mathrm{PAR}}=-\phi_{\mathrm{DYN}}$.

phase space parameters to the simulation the experimental DTL transmission could not be verified. Thus, as the error of TOF measurement of the longitudinal emittance measurement device is large, only the measured bunch length was taken into account. The values of $\epsilon_{\mathrm{z}}$ and $\alpha_{\mathrm{z}}$ were adapted such that the measured DTL transmission was reproduced.

As shown in Figure 7 and 6 both codes lead to nearly the same mean transverse emittance values behind the Alvarez DTL for different phase advance values, even the calculcated phase space distributions are in good agreement. The characteristics of the measured data is reproducible, but the simulated values are about a factor of two lower. Probably measured low energy tails are responsible for that. Besides, the beam dynamics of mismatched beams might be underestimated by the codes. The simulations confirm a minimum transverse emittance growth at a zero current phase advance of $\sigma_{0}=60^{\circ}$ while the beam brilliance is improved. Furthermore the simulations showed that the total mismatch at $\sigma_{0}=60^{\circ}$ is up to 2.6 times smaller with respect to $\sigma=35^{\circ}$. The smallest mismatch was found at $\sigma_{0}=55^{\circ}$.

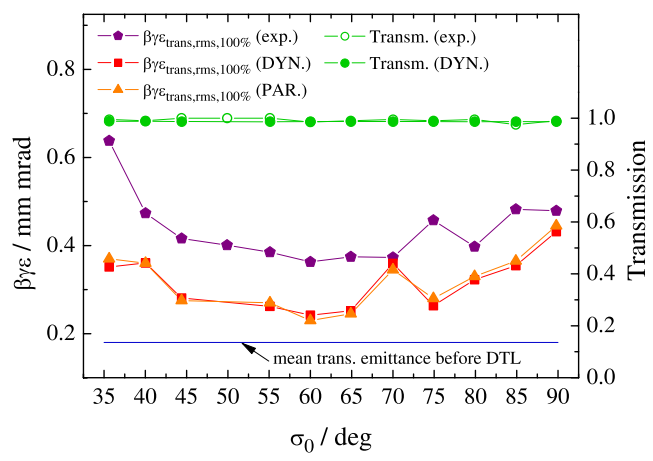

Figure 7: Measured and simulated transverse $100 \% \mathrm{rms}$ emittances behind the Alvarez DTL as function of $\sigma_{0}$.

\section{SUMMARY AND OUTLOOK}

Machine experiments at the GSI UNILAC were performed with a high intensity ${ }^{40} \mathrm{Ar}^{10+}$ beam. An improved high current matching routine based on measured emittance data was established. Three projections of the 6D phase space before the Alvarez DTL were measured as well as the transverse emittances behind the DTL for different zero current phase advances $\sigma_{0}$. It could be shown that the variation of $\sigma_{0}$ is excellent reproducible. A maximum beam brillance increase of $20 \%$ was experimentally determined at $\sigma_{0}=60^{\circ}$. For advanced studies a phase advance profile along the DTL will be investigated. Results of simulations with DYNAMION and PARMILA are in excellent agreement to each other. The minimum transverse emittance growth at $\sigma_{0}=60^{\circ}$ was confirmed, but the measured data were underestimated by a factor of 2 . As the experimental DTL transmission could not be verfied using all longitudinal emittance parameters, only the measured bunch length was used. Therefore further investigations are planned to improve the performance of the longitudinal emittance measurement device before the DTL.

\section{ACKNOWLEDGEMENT}

We acknowledge the support of the European Community-Research Infrastructure Activity under the FP6 "Structuring the European Research Area" programme (CARE, contract number RII3-CT-2003-506395).

\section{REFERENCES}

[1] W. Barth et al., Development of the UNILAC Towards a Megawatt Beam Injector, Proc. of $22^{\text {nd }}$ International LINAC Conference, Luebeck, Germany (2004), p. 246.

[2] W. Barth et al., Upgrade of the high current heavy ion UNI$L A C$ as an injector for FAIR, Nucl. Instr. and Meth. A 577 (2007) 211.

[3] P. Forck et al., Aspects of Bunch Shape Measurements for Slow, Intense Ion Beams, Proc. of $4^{\text {th }}$ DIPAC, Chester, UK (1999), p. 186.

[4] P. Forck et al., Heavy-Ion-Beam Emittance Measurements at the GSI UNILAC, Proc. of $20^{\text {th }}$ International LINAC Conference, Monterey, California, USA (2000), p. 166.

[5] W. Bayer et al., Heavy-Ion-Beam Emittance Measurements at the GSI UNILAC, Proc. of $23^{\text {rd }}$ International LINAC Conference, Knoxville, Tennessee, USA (2006), p. 177.

[6] S. Yaramyshev et al., Investigation of the Beam Matching to the GSI-Alvarez DTL Under Space Charge Conditions, Proc. of $22^{\text {nd }}$ International LINAC Conference, Luebeck, Germany (2004), p. 48.

[7] T. P. Wangler, Principles of RF Linear Accelerators, John Wiley \& Sons, Inc., New York, Chichester, Weinheim, 1998, p. 217 and 278.

[8] S. Yaramyshev et al., Development of the versatile multiparticle code DYNAMION, Nucl. Instr. and Meth. A 558 (2006) 90.

[9] H. Takeda, Parmila, LANL Report No. LA-UR-98-4478, Los Alamos, 1999 (unpublished). 